\title{
Inhibition of miR-19a-3p Increases Bortezomib-Induced Apoptosis in Myeloma Cell Lines by Targeting SOCS3
}

\author{
(1) Azam KAZEMI, (D) Saeid ABROUN, (i) Masoud SOLEIMANI \\ Department of Hematology and Blood Banking, Faculty of Medical Sciences, Tarbiat Modares University, Tehran-Iran
}

\begin{abstract}
OBJECTIVE
Apoptosis is considered the main factor inducing by bortezomib in myeloma cells. Although bortezomib (BTZ) is a crucial drug for the treatment of MM, chemoresistance is a major problem. OncomiR19a plays an oncogenic role in many types of cancer such as MM; however, the function of miR-19a in the pathogenesis of MM and drug resistance has not been identified well. The present research aims at investigating the inhibition of miR-19a by antagomir to conclude BTZ responsiveness and suggests that miR-19a may be a biomarker for the prognosis of the patient.
\end{abstract}

\section{METHODS}

In the present research, the viability and apoptosis of myeloma cells were analyzed by colorimetric MTT and Annexin-PI flow cytometric assays. QRT-PCR was implemented to evaluate the expression level of miR-19a, its targets SOCS3 and STAT3, at the mRNA level.

\section{RESULTS}

Following treating transfected cells with bortezomib we found out that miR-19a was downregulated, and the rate of apoptosis of myeloma cells after bortezomib treatment considerably increased. It indicated that mRNA of SOCS3 increased and STAT3 decreased.

\section{CONCLUSION}

The research results indicate that oncomiR-19a as a biomarker may induce better responsiveness to bortezomib in myeloma cell lines through its targets SOCS3, STAT3, and may provide new therapeutic targets in the future for myeloma. The research results indicate that oncomiR-19a as a biomarker may induce better responsiveness to bortezomib in myeloma cell lines through its targets SOCS3, STAT3 and may provide new therapeutic targets in the future for myeloma.

Keywords: Antagomir-19a; bortezomib; multiple myeloma; SOCS3.

Copyright ( 2021 , Turkish Society for Radiation Oncology

\section{Introduction}

Multiple Myeloma (MM) is some sort of tumor of differentiated B cells from the germinal center, plasma cells, within $10 \%$ of all hematologic neoplasms, and is considered the second most commonly occurring non-Hodgkin lymphoma.[1,2] Over the last two de- cades, due to advancements in available treatments, the median survival time of patients with MM has increased from 3 to 6 years and is accounting for $1.1 \%$ of total cancer deaths and the age-standardized risk Of MM is about 2\%.[3] Although great discoveries have been made in the growing evolution of new healthcare strategies in the past decade, to a great extent the tu- 
mor is still incurable and new therapies are required to overcome it.[4,5] Despite the innovation and benefitting from a new therapeutic strategy such as proteasome inhibitors, the clinical outcome of the patients aggravates, and in most patients finally, MM relapses and engenders drug resistance.[1]

The proteasome inhibitor, bortezomib (BTZ), is a crucial and an FDA-approved drug for the treatment of MM, especially relapsed \& refractory MM.[6] Although BTZ has a significant impact on the treatment of MM,[5] drug resistance or relapse are still two major challenges, and the prognosis of patients with BTZ resistance is highly undesirable.[7,8] Therefore, to prevent BTZ resistance, new therapeutic methods are desperately required. On the other hand, a more profound molecular grasp of cancer's pathogenesis is, thus, urgently required to recognize new molecular targets and to bring forth therapeutic agents that are suitable for the patient.[9] In this regard, there is developing evidence indicating that MM stems from the deregulation of noncoding RNAs, including miRNAs.[1] Recent studies show that MM is caused by interruptions in many different signaling pathways, which are driven by the microRNAs and are a class of non-coding RNAs (ncRNAs) of about 18-22 nucleotides in length. These microRNAs act as master regulators of gene expression at the post-transcriptional level via RNA interference pathways.[6] miRNAs are involved in many biological processes, including differentiation, senescence, survival, and, apoptosis. $[3,6]$ It is well established that the disturbance in the regulation of miRNAs is accompanied by the pathogenesis of diseases such as cancer, and miRNA expression profiles have prognostic implications in numerous types of cancer.[6] Altogether, miRNAs play a fundamental role as an oncogene. In other words, miRNAs operate as 'oncomiRs' if their targets are the tumor suppressor genes. [1] Therefore, controlling oncomiRs may be an effective strategy for treatment.

Chief among oncomiRs, miR-17-92 clusters that are placed in chromosome 13q31.3, including miR-19a are the very first oncomiRs that were discovered. It is proved that any disturbance in the expression level of miR-17-92 clusters results in the malignant progress of MM.[4,5] MiR-19a, a crucial component of the miR17-92 cluster, is directly involved in myeloma pathogenesis and progression of multiple myeloma.[9] Also, besides target genes of miR-19a are considered as potential biomarkers of the disease.[10] Moreover, compared with normal plasma cells, miR-19a has been revealed to be up-regulated in patients with $\mathrm{MM}$, as well as in MM cell lines.
Furthermore, miR-19a can adjust the expression of proteins that are essential for myeloma pathogenesis including suppressors of cytokine signaling (SOCSs).[9] It is reported that miR-19a targets $S O C S 3$, a potent regulator of the JAK-STAT pathway, as is followed by a considerable reduction of SOCS3 mRNA together with enhanced activation of SOCS3 target, STAT3.[9] Based on these findings, a strategy that can be developed to regulate miRNA's aberrant expression in cancer is the inhibition of up-regulated miRNAs.[1] Therefore, we have assumed that the use of mir-19a inhibitors (Antago-miR) could be a new approach for the treatment of multiple myeloma. On the other hand, based on the use of BTZ in the treatment of patients with MM and the resistance in $60 \%$ of them, the present study investigates the impact of antagomiR-19a on better responsiveness to BTZ and may present miR-19a as an effective biomarker for response to treatment. $[9,11]$

\section{Materials and Methods}

\section{Cell Lines \& Cell Culture}

In the present research, the RPMI8226 and U266 cell lines were purchased from the Pasteur Institute of Iran (IPI). The cells were grown in suspension in RPMI1640 medium (BIO-IDEA from Bio Idea Group) supplemented with $10 \%$ fetal bovine serum (FBS) (GIBCO-BRL), penicillin (100 mg/mL), streptomycin (100 $\mathrm{mg} / \mathrm{mL}$ ), and $2 \mathrm{mM}$ l-glutamine (BIO-IDEA from Bio Idea Group). Cells were maintained at $37^{\circ} \mathrm{C}$ in an environment of 5\% carbon dioxide and 95\% air and underwent passage twice a week.

\section{Reagents}

Bortezomib (PS-341) (Selleckchem.com Cat. No.S1013) was dissolved in $0.2603 \mathrm{~mL}$ DMSO to prepare a stock solution with a concentration of $50 \mathrm{mM}$ for storage at $-20^{\circ} \mathrm{C}$. LentimiRa-off-has-miR-19a-3p vector (Applied Biological Materials (ABM) Inc. with Cat. No. mh30299) with GFP promoter, miRNA insert, and kanamycin resistance gene that was transformed in DH5a E.coli strain and isolatedby Qiagen plus Midi plasmid purification kit and stored at $-20^{\circ} \mathrm{C}$.

\section{In Vitro Cell Culture and Drug Treatment}

Human myeloma cell lines RPMI8226 and U266 were cultured and $50 \mathrm{mM}$ Stock solution of Bortezomib was further diluted to working concentrations in RPMI 1640 medium before use. To determine the concentration of drug that inhibited cell proliferation by $50 \%$ (IC50), 
$5 \times 10^{3}$ cells were seeded in 96well plate, then treated with different concentration of working solutions of BTZ based on the approximate concentration that was noted in cell assay part of BTZ data-sheet in ranges of $0.5,5,50 \mu \mathrm{M}$ for U266 and 150, 450, 750, $1050 \mathrm{nM}$ for RPMI8226, mixed well in RPMI1640 media and 10\% FBS and finally incubated for 48 hours.

\section{MTT Colorimetric Assay}

Using a standard protocol, the inhibitory impact of BTZ on cell growth was assessed by 3-(4, 5-Dimethylthiazol-2-yl)-2, 5-Diphenyltetrazolium Bromide (MTT) assay. Briefly, cells from 48-hour cultures were pulsed with $10 \mu \mathrm{L}$ of $5 \mathrm{mg} / \mathrm{mL}$ MTT to each well for at least 4 hours of 48 -hour cultures, followed by $100 \mu \mathrm{L}$ of isopropanol containing $0.04 \mathrm{~N} \mathrm{HCl}$, absorbance was measured at $570 \mathrm{~nm}$ using a spectrophotometer and results were expressed as the mean of three replicates as a percentage of control (taken as 100\%). The extent of cytotoxicity was defined as the relative reduction of the optical density (OD), which correlated to the number of viable cells compare to cell control (100\%). To decide the optimum dosage of the drugs for further studies, the cell viability was plotted in a graph and the IC 50 was calculated accordingly.

\section{Cell Viability Analysis}

The effect of transfection on cellular viability was assessed by flow cytometry using propidium iodide (PI). PI can only pass through disordered areas of the dead membrane cells and intercalated with the DNA of the nuclei, emitting red fluorescence light. It was used with $1 \mathrm{mg} / \mathrm{mL}$ concentration by dissolving PI (Sigma, $\mathrm{P} 4170$ ) in $\mathrm{dH}_{2} \mathrm{O}$. PI solution was added in a final concentration of $2 \mu \mathrm{g} / \mathrm{mL}$ to $1 \times 10^{6}$ cells in suspension, incubated at a dark place and after $5 \mathrm{~min}$ utes, analyzed by flow cytometry using Attune NXT flow cytometer.

\section{Analysis of Apoptosis}

The apoptosis of cell lines after transfection with Plenti-III-miR-Off -has-miR-19a -3p vector, after treatment with bortezomib and after treatment of transfected cells with bortezomib compared with untreated cells were analyzed with Annexin V/PI (eBioscience Cat. No. 88-8005 from Thermo Fisher Scientific) by flow cytometry using Attune NXT flow cytometer. Cells were washed in PBS and then they were washed in $1 \mathrm{X}$ bindings buffer once, after that we resuspended cells in $1 \mathrm{X}$ bindings buffer at $1 \times 10^{6} / \mathrm{mL}$, then we added 5 $\mu \mathrm{L}$ of FITC-conjugated Annexin V to $100 \mu \mathrm{L}$ of the cell suspension, incubated for 10-15 minutes in a dark place at room temperature, then they were washed with binding buffer, resuspended in it afterward we added $5 \mu \mathrm{L}$ of Propidium Iodide Staining Solution (Sigma, P 4170), analyzed by flow cytometry.

\section{Prediction of SOCS3 as a Target of miR-19a}

Target Scan (Version 5) and pic tar were used to confirm the SOCS3 molecule as a target of miR-19a in its 3' UTR. Target Scan predicted biological targets of miRNA by searching for the presence of conserved 8 and 7 base sites that match its seed region.

\section{Cell Transfection}

Cells were grown in RPMI1640 medium containing no antibiotics before transfection. U266 and RPMI8226 were transfected by final concentration $2 \mu \mathrm{g}$ of Plenti-III-miR-Off-has-miR-19a $-3 p$ vector containing GFP purchased from Applied Biological Materials Inc. (ABM). Transfection of cells was performed using UltraCruz ${ }^{\oplus}$ Transfection Reagent (SCBT, Santa Cruz Biotechnology, Inc.). Briefly, before transfection, we prepared a transfection reagent and vector in Opti-MEM I reduced serum medium (Gibco) following the manufacturer's protocol. After preparing the transfection reagent and plasmid in OPTI-MEM I medium and incubation at room temperature, plasmid reagent was added to transfection reagent, vortex vigorously incubates for 20 minutes. $6 \times 10^{3}$ cells were added to the Eppendorf tube and were poured above mix dropwise to the cells, incubated in the incubator for 2 hours, every half an hour flicked with a fingertip, finally were transferred to 6-well plate and after 24-72 hours GFP expression was evaluated by Attune NXT flow cytometer.

\section{Q-RT-PCR Assessment of miR-19a Expression}

Total RNA was isolated from un-transfected, transfected and BTZ treated cells: RPMI8226 and U266 while following the Trizol manufacturer's protocol (InvitrogenTM, USA). 2000ng of RNA was reverse transcribed using specific microRNA stem-loop primers from Qiagen for miR-19a, to generate cDNA using Hyperscript reverse transcriptase first-strand synthesis kit (GeneAll Biotechnology Co., Ltd, Korea). The expression of Snord47 was used as the internal control for miRNA expression normalization. The quantitative real-time PCR was performed with the SYBR ${ }^{\oplus}$ Premix Ex Taq ${ }^{\text {Tw }}$ miRNA RT-qPCR Detection Kit (Takara, Cat.No. RR820Q) is using the Qiagen Rotor-Gene Q 5PLEX HRM Real-Time PCR and primers with sequences 


\begin{tabular}{|c|c|c|c|}
\hline Gene & Primer (5'-3') & Product length (bp) & $\begin{array}{c}\text { Annealing } \\
\text { temperature }\left({ }^{\circ} \mathrm{C}\right)\end{array}$ \\
\hline \multirow[t]{4}{*}{ miR-19a } & F: CTC CGT TGT CCA AAT CTA TG & 72 & 60 \\
\hline & R: GAG CAG GGT CCG AGG T & & \\
\hline & RT: GTC GTA TGC AGA GCA GGG TCC GAG & & \\
\hline & GTA TTC GCA CTG CAT ACG ACT CAG TT & & \\
\hline \multirow[t]{4}{*}{ Snord 47} & F: ATC ACT GTA AAA CCG TTC CA & 70 & 60 \\
\hline & R: GAG CAG GGT CCG AGG T & & \\
\hline & RT: GTC GTA TGC AGA GCA GGG TCC GAG & & \\
\hline & GTA TTC GCA CTG CAT ACGACA ACC TC & & \\
\hline \multirow[t]{2}{*}{ SOCS3 } & F: CCAAGGACGGAGACTTCG & 140 & 60 \\
\hline & R: GAAACTTGCTGTGGGTGAC & & \\
\hline \multirow[t]{4}{*}{ STAT3 } & F: CGG CGT CCA GTT CAC TAC & 97 & 60 \\
\hline & R: CCC AGA GTC TTT GTC AAT GC & & \\
\hline & R: CCT CTG GTC CTG GTA TGA AGA ATG & & \\
\hline & R: CAG GAG AAA TCA ACA GAGGC & & \\
\hline
\end{tabular}

matching with table 1 . PCR program cycling parameters included $95^{\circ}$ for $15 \mathrm{~s}, 58^{\circ}$ for the $30 \mathrm{~s}, 72^{\circ}$ for $30 \mathrm{~s}$ for 45 cycles. Data analysis was done by $2-\Delta \Delta$ CT to calculate the fold change of miR-19a relative expression compared to untreated control.

\section{Quantitative RT-PCR Analysis for SOCS3, STAT3 Genes}

Total RNA was isolated from un-transfected, transfected and BTZ treated cells: RPMI8226 and U266 following the Trizol manufacturer's protocol (InvitrogenTM, USA). $2 \mu \mathrm{g}$ of total RNA was reverse transcribed into cDNA using Hyperscript reverse transcriptase firststrand synthesis kit with oligo-dT primers following the manufacturer's instructions (GeneAll Biotechnology Co., Ltd, Korea) for evaluation of SOCS3, STAT3 genes. The expression of $\beta$-Actin was used as an internal control. The quantitative real-time PCR was performed with the SYBR ${ }^{\oplus}$ Premix Ex Taq ${ }^{\text {Tw }}$ miRNA RT-qPCR Detection Kit (Takara, Cat. No. RR820Q) using the Qiagen Rotor-Gene Q 5PLEX HRM Real-Time PCR. PCR program cycling parameters included $95^{\circ}$ for $15 \mathrm{~s}, 58^{\circ}$ for the $30 \mathrm{~s}, 72^{\circ}$ for $30 \mathrm{~s}$ for 45 cycles. Data analysis was performed by $2-\Delta \Delta \mathrm{CT}$ to calculate the fold change of the above genes' relative expression compared to untreated control.

\section{Statistical Analysis}

Data were presented as mean \pm standard deviation. The statistical analysis was performed with the GraphPad
Prism 8.4.0 software The mean values of two groups or multiple groups were compared by one-way analysis of variance (ANOVA). $\mathrm{P}<0.05$ was considered statistically significant. Flow cytometric assays were analyzed with FlowJo version 7.6.1.

\section{Results}

IC50 of BTZ in (U266 and RPMI 8226) MM Cell Lines was $5 \mu \mathrm{M}$ and $150 \mathrm{nM}$, Respectively

We treated RPMI 8226 and U266 cell lines with different concentrations of BTZ to optimize its IC50 for the treatment of cell lines with proper concentrations throughout the analysis. By using the MTT assay and viability test with PI through flow cytometry, we found out that the optimized concentration of BTZ for the U266 cell line was $5 \mu \mathrm{M}$ and $150 \mathrm{nM}$ for RPMI8226 (Fig. 1a, c, and b, d).

\section{Downregulation of miR-19a Decreased Cell Viabil-} ity and Prevented the Proliferation of Myeloma Cell Lines

We first evaluated the expression level of miR-19a in non-transfected (control) myeloma cell lines (RPMI 8226 and U266). After the transfection of myeloma cell lines with the LentimiRa-off-has-mir-19a-3p vector, the efficiency of transfection was monitored by GFP evaluation using both fluorescent microscopy (Fig. $2 \mathrm{a}$ and $3 \mathrm{a}$ ) and flow cytometry (Fig. $2 \mathrm{~b}$ and $3 \mathrm{~b}$ ). The expression level of miR-19a was then determined in 


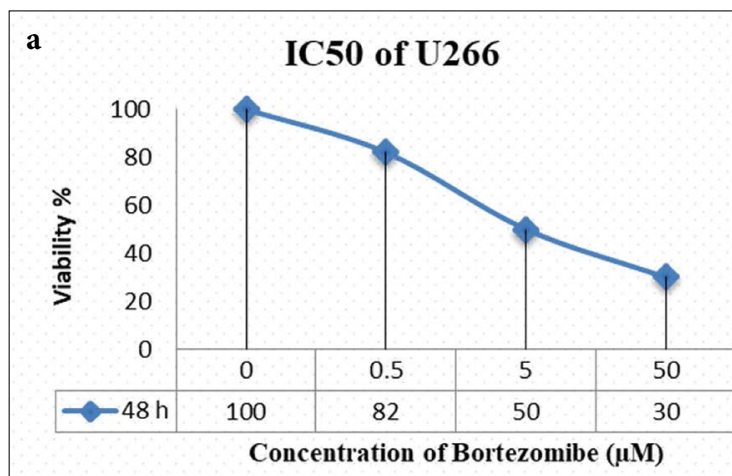

c
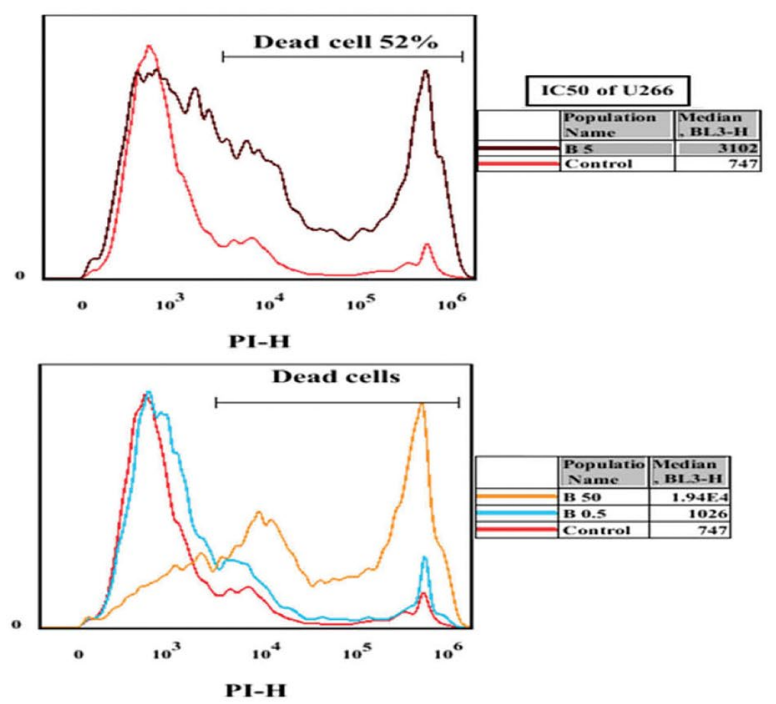

b

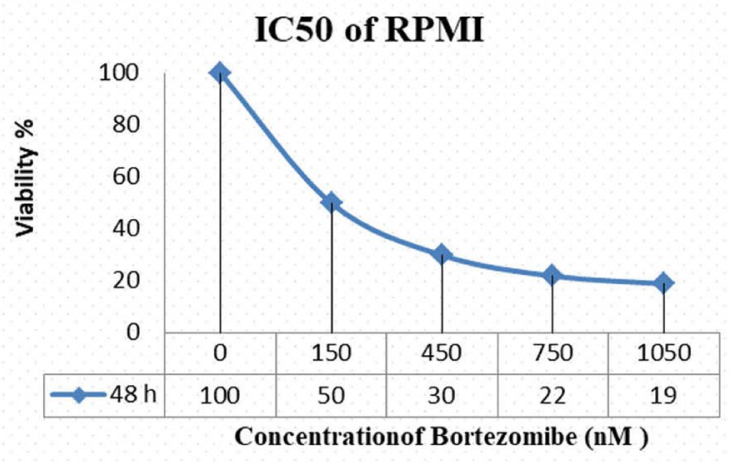

d
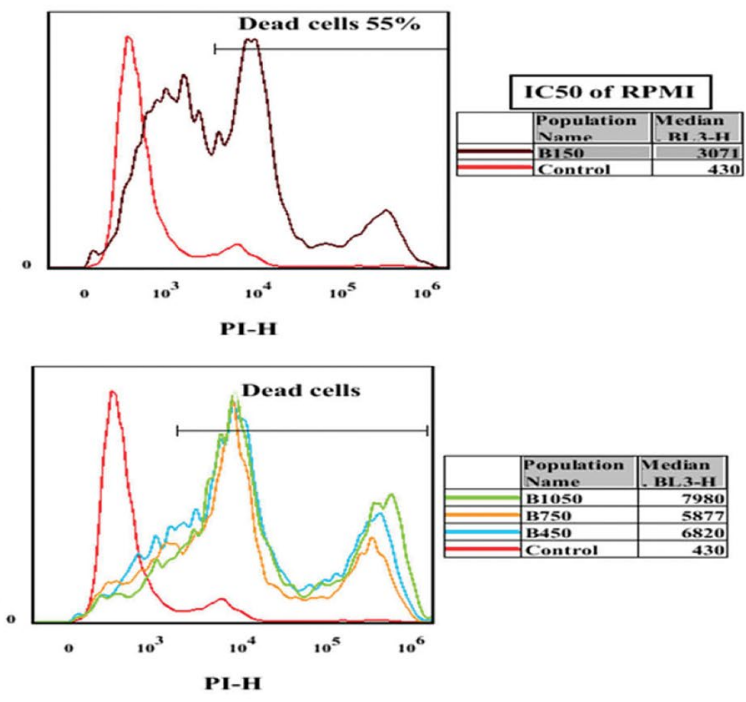

Fig. 1. IC50 evaluation of U266 and RPMI 8226 cell lines to optimize concentration of BTZ. (a, b) U266 and RPMI 8226 cell lines were incubated with increasing concentrations of BTZ ( 0 to $50 \mu \mathrm{M})$ and ( 0 to $1050 \mathrm{nM})$ respectively for 48 hours and cell viability was assessed by MTT colorimetric assay. (c, d) U266 and RPMI 8226 cells after treatment were incubated with Propidium iodide (PI) and the percentage of dead cells was assessed by flowcytometry.

transfected myeloma cell lines (RPMI 8226 and U266) by qRT-PCR. As shown in fig. 2C and 3C, compared with the un-transfected myeloma cell line, the expression level of miR-19a significantly decreased in the transfected group. The data showed that antago-miR19a downregulated expression of miR-19a which is a previously-known oncomiR. After the transfection of myeloma cell lines (RPMI 8226, U266) with LentimiRa-off-has-mir-19a-3p vector, cell viability was evaluated in 72 hours with PI (Propidium Iodide) and the rate of viability was detected by flow cytometry (Fig. $2 \mathrm{~d}$ and $3 \mathrm{~d}$ ). The data confirms the possibility of the analyses of transfected cell lines within 72 hours after transfection, while indicating that the viability of cell lines decreased because of the downregulation of miR19a. A comparison with the negative control group, suggests that the miR19a suppression decreased the proliferation of the cells.

\section{The Expression Level of miR-19a in Myeloma Cell Lines was Reduced Upon BTZ Treatment}

The expression level of miR-19a was evaluated after BTZ treatment by qRT-PCR analysis, and our data showed that its expression level was compared with non-treated cell lines, reduced after treatment. In the meantime, this expression was also evaluated in cells transfected by the LentimiRa-off-has-mir-19a-3p vector and the data showed that miR-19a expression level was highly reduced after BTZ treatment, compared to non-treated and non-transfected controls (Fig. $2 \mathrm{c}$ and $3 c$ ). Cell viability evaluation in 72 hours with Propidium Iodide (PI) using flow cytometry showed that it is 
a
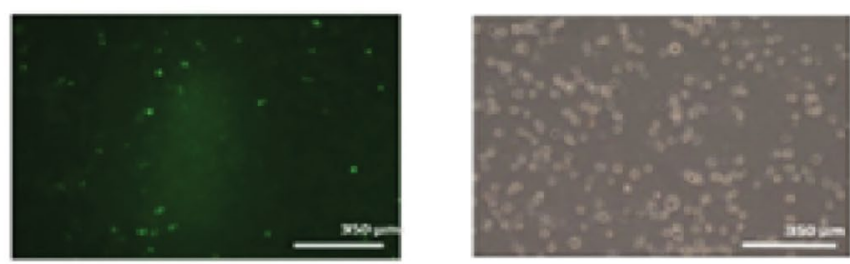

c

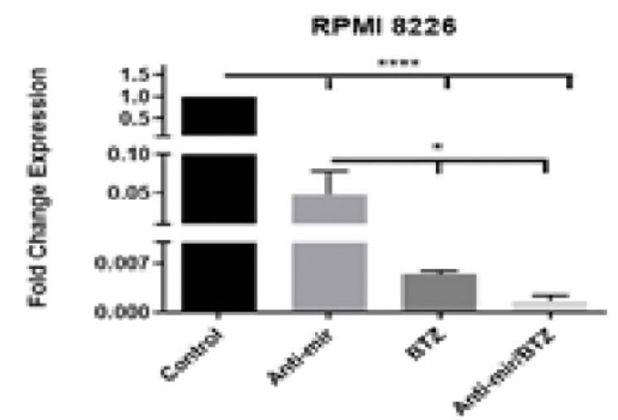

b

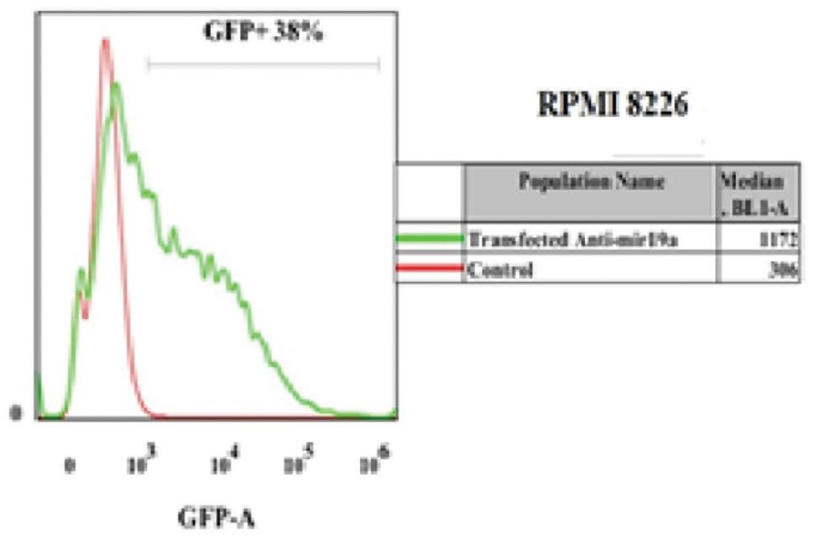

d

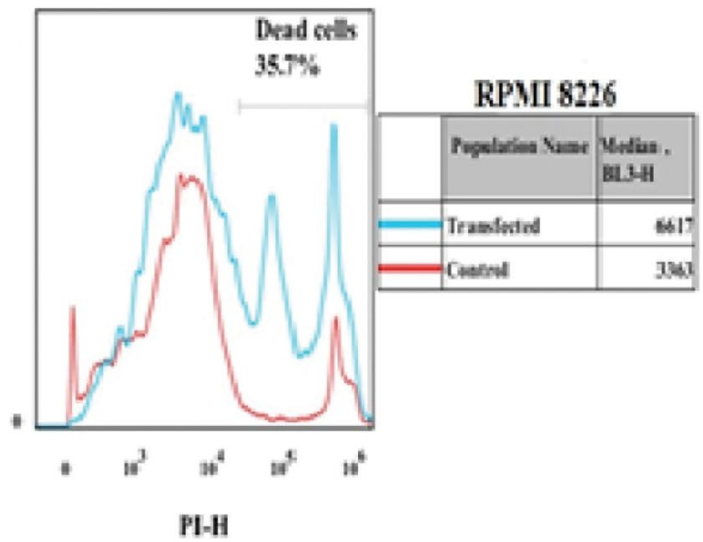

Fig. 2. RPMI 8226 cells were transfected with the lentiviral-Anti-mir-19a vector. (a, b) The transfection efficiency was assessed by fluorescence microscopy and flowcytometry through GFP green fluorescent. (c) The expression of miR-19a was assessed by qRT-PCR in non-transfected RPMI cell lines (control), transfected RPMI cell lines with lentiviral-Anti-mir19a vector, treated RPMI cell lines with BTZ and treated RPMI cell lines with BTZ after transfection with lentiviral-Anti-mir19a vector. The ratios of miR-19a were calculated relative to snord47. Values are expressed as the mean \pm standard deviation of 3 independent experiments. ${ }^{* *} \mathrm{P}$ value $<0.001{ }^{*} \mathrm{P}$ value $<0.05$ vs. the control. (d) Evaluation of Myeloma cell lines RPMI 8226 viability 72 hours after transfection. After transfection of RPMI with Plenti-Anti-mir19a vector cells were incubated with Propidium iodide (PI) and cell viability assessed by flow cytometry after $72 \mathrm{~h}$.

possible to evaluate every analysis on transfected cell lines in 72 hours after transfection and on the other hand, the viability of cell lines decreased due to the downregulation of miR-19a (Fig. 2d and 3d).

\section{Anti-miR-19a Increased Susceptibility to Borte- zomib-Induced Apoptosis}

In order to investigate that antago-miR-19a affects drug-induced apoptosis of myeloma cells, the percentage of apoptotic cells by Annexin -V, PI through flow cytometry after transfection of myeloma cell lines by lentimiRa-off-has-mir-19a-3p and incubation with bortezomib (BTZ, final concentration $5 \mu \mathrm{M}$ for U266 and $150 \mathrm{nM}$ for RPMI8226) for 48 hours were analyzed. Analysis of flow cytometry data showed that the percentage of cells that underwent apoptosis increased after transfection, compared with non-transfected cell lines and the negative control (Fig. 4a, b, c and d) (RPMI8226: 23.5\% Vs. $68.2 \mathrm{p}=0.0038$ U266: 25.2 vs. $96.4 \mathrm{p}=0.0006)$. The data showed that antago-miR-19a could increase myeloma cell susceptibility to drug-induced apoptosis.

SOCS3 and STAT3 mRNA expression level upregulated and downregulated, respectively after transfection of myeloma cell lines with LentimiRa-off-has-mir$19 \mathrm{a}-3 \mathrm{p}$ vector.

After recognizing SOCS3 as the target of miR-19a, SOCS3 and its target STAT3 mRNA level in myeloma cell lines after transfection with Lentini-off-has-mir$19 a-3 p$ vector was evaluated. The data showed that the mRNA expression level of SOCS3 increased, while STAT3 mRNA expression level decreased, compared with non-transfected cell lines as the negative control (Fig. 5a, b, 5c, and d). 


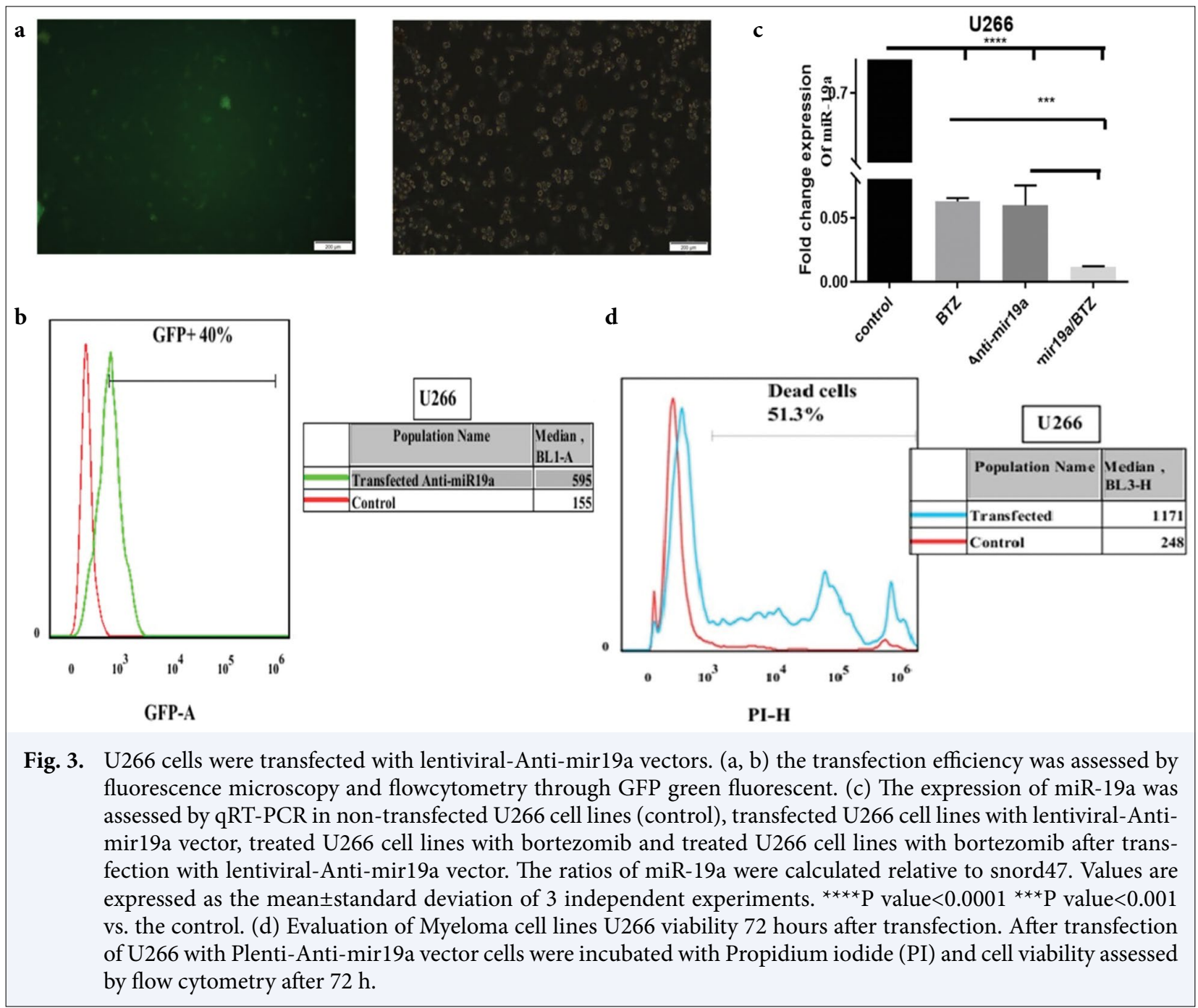

\section{Discussion}

Based on several findings of the studies previously conducted,[12] the first outcome of deregulated miRNA expression in MM, was reported by Loffler et al. in 2007,[13] which demonstrated miR-21 ectopic expression makes MM cells independent from the IL-6 growth stimulus. Pichiorri et al. identified a miRNA signature associated with the transformation of normal PCs to clinical MM via MGUS and demonstrated miR-32, miR-21, miR-17-92, miR-106b-25 cluster, and $\mathrm{miR}-181 \mathrm{a} / \mathrm{b}$ were upregulated in both $\mathrm{MM}$ cell lines and primary tumors versus normal PCs: among them miR-17-92 cluster were highly expressed only in MM patients.[14] OncomiR-19a, as one of the members of miR-17-92 clusters has been proved to be involved in the promotion of cell proliferation, migration, and deduction of apoptosis, and suggests a critical role for it in the pathogenesis of myeloma.[10] Considering that better response in patients with the low level of miR-19a in serum and shortened progression-free with downregulation of it obtained from others studies,[15] we tried to evaluate the responsiveness of MM cell lines to BTZ in the presence of antagomiR-19a to find a way to evade from resistance to it through targets of miR-19a. Recent studies figured out that SOCS3 is a target of miR-19a and indicated it is a negative regulator of SOCS3 $[16,17]$ and showed that the molecule beneath it, i.e. STAT3, is a significant promoter of cancers such as MM when activated. $[5,18,19]$ On the other hand STAT3, one of the components of the JAK/STAT pathway acts as an oncogene in human cancers.[19,20] Some miRNAs have close relativity with drug resistance, for example, past studies have shown that miR-181a expression is consistent with MM tumor load and could be a biomarker 


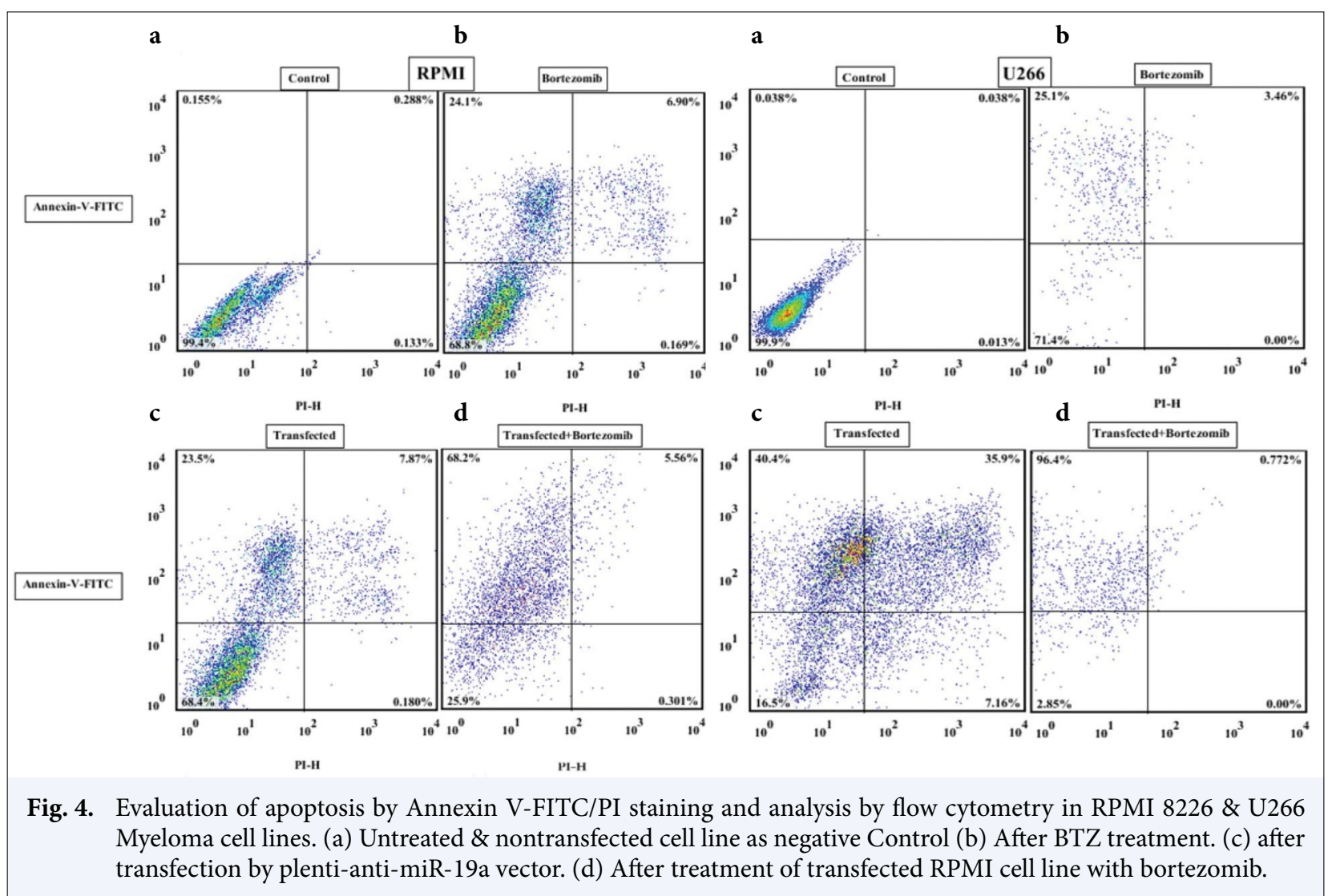

for treatment monitoring as much as miR-20a that showed the potential of a diagnostic biomarker.[4,6] $\mathrm{BTZ}$ as a proteasome inhibitor is an effective drug in the treatment of MM; however, drug resistance is still a major problem in $60 \%$ of patients. $[8,21]$ So our data primarily showed that antagomir-19a down-regulated the miR-19a expression, and BTZ caused inhibition of miR-19a in a concentration-dependent manner and thereby in the presence of antago-miR-19a and BTZ, miR-19a efficiently downregulated in parallel with an increase in the proportion of apoptotic cells following treatment with BTZ. On the other hand, our viability studies showed that the ratio of the proliferation of myeloma cell lines decreased after transfection with antagomiR-19a vector. The data are consistent with other studies that proved miR-181a and miR-20a inhibited by BTZ.[6] In another part of our studies, we found out that downregulation of STAT3 and upregulation of SOCS3 at mRNA level which confirmed the reality of miR-19a function on SOCS3 is a negative regulator, the same as previous data.[16,17] As much as, the oncogenic function of STAT3 has been previously proved, $[18,19]$ we could conclude that inhibition of miR-19a causes suppression of STAT3; and as indicated in our results, the expression level of STAT3 in BTZ treatment highly decreased using it in parallel with anatago-miR-19a. So, it can be argued that miR19a inhibition approach could be used to improve BTZ responsiveness in myeloma cells.

The results are honorable \& supplement the findings that microRNAs are differentially expressed in BTZ-resistant myeloma cells. So miR-19a could be proposed as a prognostic biomarker for responsiveness to BTZ in MM patients. To overcome the resistance and improve the level of responsiveness to BTZ, miR-19a targets such as SOCS3 and STAT3 could be tracked. We have faced some restrictions in our studies that must be adverted. They include the use of other MM cell lines and myeloma cells obtained from MM patients, and the evaluation of targets in protein level expression and downstream molecules, that could have helped us to generalize our results.

\section{Conclusion}

Overall, our data propose that induction of antago-miR-19a in myeloma cell lines resulted in a downregulation of miR-19a and enhancement of the level of 


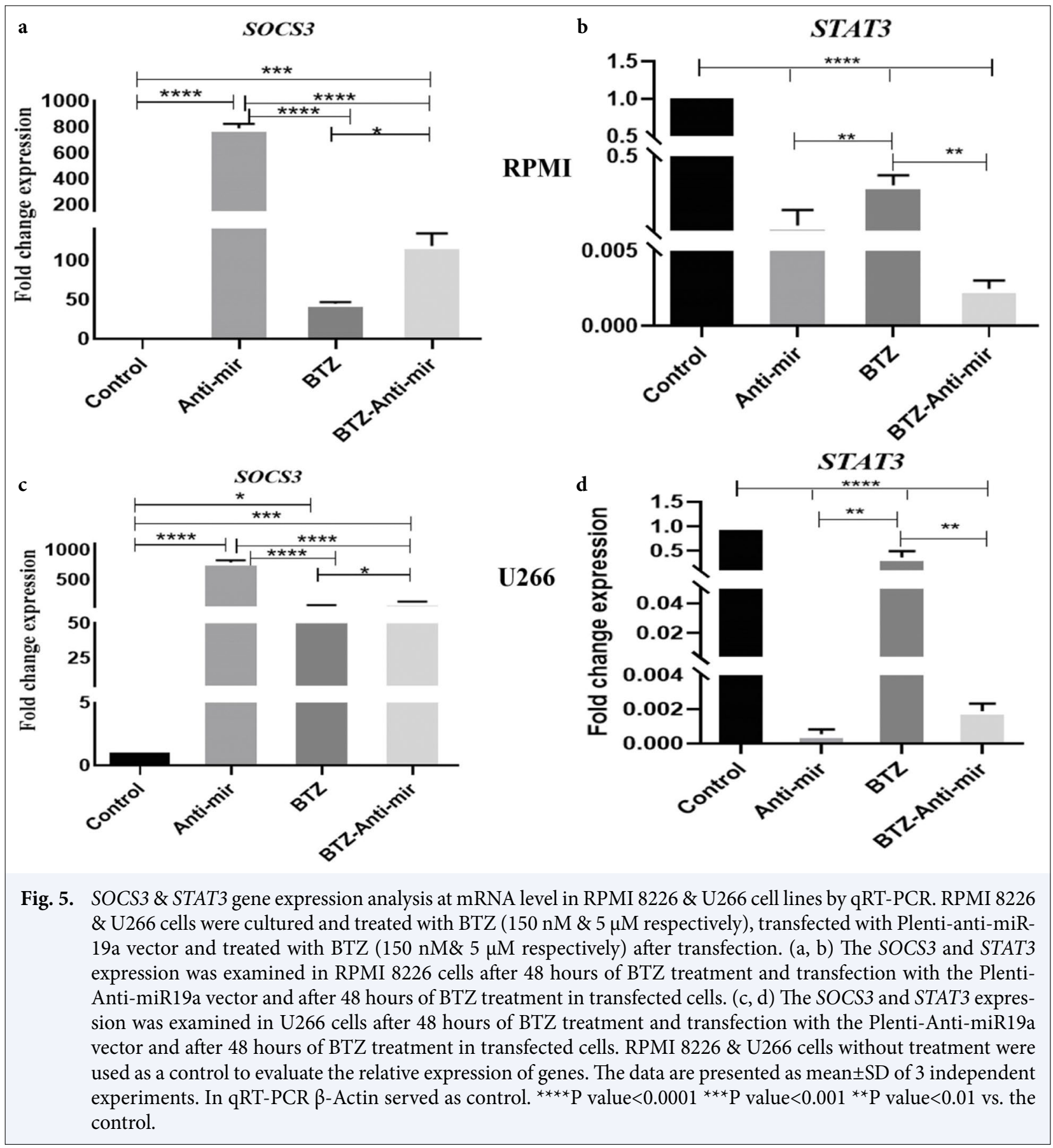

responsiveness to BTZ treatment. On the other hand, the ratio of apoptosis in BTZ-treated cell lines was drastically more effective in the presence of miR-19a inhibition, so miR-19a could be suggested as a prognostic biomarker in MM treatment.

Peer-review: Externally peer-reviewed.

\section{References}

1. Caracciolo D, Montesano M, Altomare E, Scionti F, Di Martino MT, Tagliaferri P, et al. The potential role of miRNAs in multiple myeloma therapy. Expert Rev Hematol 2018;11(10):793-803.

2. Szymczyk A, Macheta A, Podhorecka M. Abnormal microRNA expression in the course of hematological malignancies. Cancer Manag Res 2018;10:4267-77. 
3. Bray F, Ferlay J, Soerjomataram I, Siegel RL, Torre LA, Jemal A. Global cancer statistics 2018: GLOBOCAN estimates of incidence and mortality worldwide for 36 cancers in 185 countries. CA Cancer J Clin 2018;68(6):394-424.

4. Zhang X, Chen Y, Zhao P, Zang L, Zhang Z, Wang X. MicroRNA-19a functions as an oncogene by regulating PTEN/AKT/pAKT pathway in myeloma. Leukemia \& lymphoma 2017;58(4):932-40.

5. Yin Z, Yang J, Ning R, Liu Y, Feng M, Gu C, et al. Signal pathways, diseases, and functions associated with the miR-19a/92a cluster and the use of berberine to modulate the expression of this cluster in multiple myeloma cells. J Biochem Mol Toxicol 2018;32(6):e22057

6. Peng J, Thakur A, Zhang S, Dong Y, Wang X, Yuan $\mathrm{R}$, et al. Expressions of miR-181a and miR-20a in RPMI8226 cell line and their potential as biomarkers for multiple myeloma. Tumor Biol 2015;36(11):854552.

7. Richardson PG, Barlogie B, Berenson J, Singhal S, Jagannath $\mathrm{S}$, Irwin $\mathrm{D}$, et al. A phase 2 study of bortezomib in relapsed, refractory myeloma. $\mathrm{N}$ Engl J Med 2003;348(26):2609-17.

8. Niewerth D, Jansen G, Assaraf YG, Zweegman S, Kaspers GJ, Cloos J. Molecular basis of resistance to proteasome inhibitors in hematological malignancies. Drug Resist Updat 2015;18:18-35.

9. Lv H, Wu X, Ma G, Sun L, Meng J, Song X, et al. An integrated bioinformatical analysis of miR-19a target genes in multiple myeloma. Exp Ther Med 2017;14(5):4711-20.

10. Wang Y, Zhao S, Zhu L, Zhang Q, Ren Y. MiR-19a negatively regulated the expression of PTEN and promoted the growth of ovarian cancer cells. Gene 2018;670:166-73.

11. Zhu B, Ju S, Chu H, Shen X, Zhang Y, Luo X, et al. The potential function of microRNAs as biomarkers and therapeutic targets in multiple myeloma. Oncol Lett
2018;15(5):6094-106.

12. Peng Y, Croce CM. The role of MicroRNAs in human cancer. Signal Transduct Target Ther. 2016 Jan 28;1:15004.

13.Loffler D, Brocke-Heidrich K, Pfeifer G, Stocsits C, Hackermuller J, Kretzschmar A K, et al. Interleukin-6 dependent survival of multiple myeloma cells involves the STAT3-mediated induction of microRNA-21 through a highly conserved enhancer. Blood 2007;110(4):1330-33.

14. Pichiorri F, Suh S S, Ladetto M, Kuehl M, Palumbo T, Drandi D, et al. MicroRNAs regulate critical genes associated with multiple myeloma pathogenesis. Proc Natl Acad Sci USA 2008;105(35):12885-90.

15. Federico C, Sacco A, Belotti A, Ribolla R, Cancelli V, Giacomini A, et al. Circulating microRNAs and their role in multiple myeloma. Non-coding RNA 2019;5(2):37.

16. Collins AS, McCoy CE, Lloyd AT, O’Farrelly C, Stevenson N J. miR-19a: an effective regulator of SOCS3 and enhancer of JAK-STAT signaling. PLoS One 2013;8(7):e69090.

17. Mahony R, Ahmed S, Diskin C, Stevenson N J. SOCS3 revisited: a broad regulator of disease, now ready for therapeutic use? Cell Mol Life Sci 2016;73(17):3323-36.

18. He G, Karin M. NF-kappaB, and STAT3 - key players in liver inflammation and cancer. Cell Res 2011;21(1):159-68.

19. Bromberg J. Stat proteins and oncogenesis. J Clin Invest 2002;109(9):1139-42.

20. Selvi N, Kaymaz BT, Gunduz C, Aktan C, Kiper H D, Sahin F, et al. Bortezomib induces apoptosis by interacting with the JAK/STAT pathway in K562 leukemic cells. Tumour Biol 2014;35(8):7861-70.

21. Rastgoo N, Abdi J, Hou J, Chang H. Role of epigenetics-microRNA axis in drug resistance of multiple myeloma. J Hematol Oncol 2017;10(1):121. 